\title{
Form invariance of the moment sum-rules for jellium with the addition of short-range terms in the pair-potential
}

\author{
Riccardo Fantoni ${ }^{1, *}$ \\ ${ }^{1}$ Università di Trieste, Dipartimento di Fisica, \\ strada Costiera 11, 34151 Grignano (Trieste), Italy
}

(Dated: December 3, 2019)

\begin{abstract}
We find the first three (even) structure factor moments for a (non-quantum) one-component Jellium made of particles living in three dimensions and interacting with a Coulomb pair-potential plus a short-range term with either a finite range or decaying exponentially fast at large distances. Starting from the hierarchical Born-Green-Yvon equations we show that they are all form invariant respect to the addition of the short-range term. We discuss the relevance of the present study to interpret the failure of the moment sum-rules of ionic-liquids at criticality.

PACS numbers: 05.70.Fh, 61.20.Qg, 64.60.F-, 64.70.F-

Keywords: sum-rule, multipolar sum-rule, structure factor moments, internal screening, external screening, one-component plasma, Jellium, short-range interaction
\end{abstract}

\footnotetext{
*rfantoni@ts.infn.it
} 


\section{INTRODUCTION}

A prototypical model of solid state physics describing free electrons in metallic elements is the one-component Jellium: a statistical mechanics one-component fluid of point-wise charged particles made thermodynamically stable by the addition of a uniform inert neutralizing background. This fluid has been studied in great details in history both in its classical and in its quantum versions. Here we will only deal with the classical version of the model. In particular several plausible exact relationships between the $n$-point correlations functions, the so called sum-rules, has been determined over the years [1]. Of particular interest, due to the direct link with scattering experiments on the fluid, are the even moments of the structure factor, the so called moment sum-rules, which give the coefficients of the even powers of the wavenumber in a large wavelength expansion of the structure factor. The zeroth-moment sum-rule, or charge sum-rule, is commonly known as a consequence of the internal screening properties of the Coulomb system and has been known since the work of Debye and Hückel [2]. The second-moment or Stillinger-Lovett [3] sum-rule is due to the external screening and has been proved rigorously for the first time by Martin et al. [4]. The fourth-moment sum-rule has been proved rigorously for the first time by Vieillefosse [5] after it had been established earlier with various heuristic arguments [6 8] .

A mixture of charged particles can have species with opposite charges. In these cases in addition to the electrical neutrality of the system with the introduction of a neutralizing background it is necessary to introduce a hard-core on the particles, in order to assure thermodynamical stability. It is then important to understand how the addition of a shortrange regularizing term (with compact support or decaying exponentially fast [9]) to the pure Coulomb pair-potential influences the various sum-rules.

In this work we perform this study on the one-component Jellium extending Vieillefosse [5] work to a pair-potential where we add to the Coulomb term a generic short-range term with either a finite support or exponentially decaying at large distances. We work in three spatial dimensions leaving the extension to other dimensions, to a mixture, and to more general short-range potential regularizations to future works. We start from the constituent Born-Green-Yvon hierarchic equations [10] for the $n$-point correlation functions and with certain assumptions on the decay of the $n$-particle Ursell functions as subgroups of particles are infinitely separated (the exponential clustering hypothesis) we use a series of multipolar 
sum-rules [1] to determine the first three even structure factor moments.

We already know that the forms of the first two even moments are not influenced by the presence of the regularizing short-range term in the pair-potential [1, 4]. We will find that also the fourth-moment is form invariant.

This work is a step forward in the understanding of the failure of the second and fourth moment sum-rules recently observed in the restricted primitive model (RPM) at criticality [11, 12]. In fact, it was only until recently that the previously unknown form of the fourth moment sum-rule for the RPM was established using a semi-heuristic argument [13, 14] claiming form invariance respect to the pure coulombic case. Our present result gives a rigorous first principle proof of the form invariance at least in the weak coupling regime and for the one-component Jellium. This fact, if confirmed for the two-component plasma, relegates the failure of the sum-rules at criticality to the disruption, upon approaching a phase transition, of the exponential clustering, i.e. the decay to zero of the truncated correlations faster than any inverse power of distance as groups of particles are separated by an infinite distance. In fact, it has to be expected that at criticality the correlation functions develops long-range tails with monotonous or oscillating inverse power law decay [15].

The work is organized as follows: In section 2 we find again the zeroth moment sumrule following the original derivation of Martin et al. [1], in section 3 we find again the second moment sum-rule following the original derivation of Martin et al. [1, 4], in section 4 we derive the fourth moment sum-rule with a route alternative to the one of Vieillefosse [5] which explains clearly from the point of view of the BGY why also this moment is form invariant upon the addition of a short-range term to the Coulomb pair-potential, in section 5 we determine the isothermal compressibility of the system, and section [ is for the concluding remarks. 


\section{DERIVATION OF THE ZEROTH MOMENT SUM-RULE}

The second order Born-Green-Yvon (BGY) hierarchy [1]

$$
\begin{aligned}
\nabla_{\mathbf{r}_{1}} u_{2}(1,2)= & \beta \mathbf{F}_{21}\left[u_{2}(1,2)+1\right]+ \\
& \rho \int d \mathbf{r}_{3}\left[1+u_{2}(1,2)+u_{2}(1,3)+u_{2}(2,3)+u_{3}(1,2,3)\right] \beta \mathbf{F}_{31}- \\
& \rho \int d \mathbf{r}_{3}\left[u_{2}(1,2)+1\right] \beta \mathbf{F}_{31} \\
= & \beta \mathbf{F}_{21}\left[u_{2}(1,2)+1\right]+\rho \int d \mathbf{r}_{3}\left[u_{2}(2,3)+u_{3}(1,2,3)\right] \beta \mathbf{F}_{31}
\end{aligned}
$$

where $\beta=1 / k_{B} T$ with $k_{B}$ Boltzmann's constant and $T$ the absolute temperature, $\rho$ is the density of the fluid, $\mathbf{F}_{21}=-\boldsymbol{\nabla}_{\mathbf{r}_{1}} v(1,2)$, with $v(1,2)$ the pair-potential that is the sum of a Coulomb term $v^{c}(1,2)=e^{2} /\left|\mathbf{r}_{2}-\mathbf{r}_{1}\right|$ and a short-range term $v^{s r}(1,2)$ with compact support or decaying exponentially fast [9]. We will also call $\mathbf{F}_{21}^{c}=-\boldsymbol{\nabla}_{\mathbf{r}_{1}} v^{c}(1,2)$ and $\mathbf{F}_{21}^{s r}=-\nabla_{\mathbf{r}_{1}} v^{s r}(1,2)$. According to Ref. [9] the Ursell functions $u_{n}(1,2, \ldots, n)$ must satisfy exponential clustering [1], i.e. they should tend to zero (monotonously or oscillating) faster than any inverse power of the distance as the distance between any group of particles at $\left(\mathbf{r}_{1}, \mathbf{r}_{2}, \ldots, \mathbf{r}_{n}\right)$ tends to infinity. The Ursell functions are assumed to depend only on the shape of the figure formed by the various points (and not on its space orientation) and they are symmetrical in any permutation of the particles. The first assumption is a consequence of the homogeneity and isotropy of the fluid, the second is a consequence of distinguishability of the particles. Of course the exponential clustering assumption is valid for the high temperature (low density) infinite homogeneous phase of the fluid when the correlation functions are believed to obey to the BGY hierarchy. We will generally indicate vectors with a bold-face letter and absolute values of vectors with a normal (Roman) version of the same font $r=|\mathbf{r}|$. We use a hat to denote the unit vector $\hat{\mathbf{r}}=\mathbf{r} / r$.

In the second equality of Eq. (1) we used the fact that $\int d \mathbf{r}_{3} u_{2}(1,3) \mathbf{F}_{13}=0$ by symmetry. Now we observe that the left hand side of Eq. (1) tends to zero faster than any inverse power of $x=|\mathbf{x}|=\left|\mathbf{r}_{2}-\mathbf{r}_{1}\right|$ as $x$ tends to infinity and the same is true for the first and fourth terms on the right hand side. So the sum of the second and third terms on the right hand side must vanish in the same way, in this limit. Then we require that

$$
\int d \mathbf{r}_{3} s(2,3) \mathbf{F}_{31}
$$


where $s(2,3)=\rho u_{2}(2,3)+\delta(2,3)$ and $\delta$ is the Dirac delta function, tends to zero faster than any power of the distance $x$ when the latter tends to infinity. Expanding Eq. (2) in powers of $1 / x$ in this limit, we deduce

$$
\begin{gathered}
\int d \mathbf{r}_{3} s(y) y^{l} P_{l}(\hat{\mathbf{x}} \cdot \hat{\mathbf{y}})=0, \quad l \geq 1 \\
I_{0}=\int d \mathbf{r}_{3} s(y)=0
\end{gathered}
$$

where $\mathbf{y}=\mathbf{r}_{3}-\mathbf{r}_{2}$ and $P_{l}$ are the Legendre polynomials. Eq. (4) is the zeroth moment sum-rule also known as the charge or electroneutrality sum-rule [1]. It is the simpler of the multipolar sum-rules (3). We immediately see that in our derivation we did not use the fact that $v$ is purely Coulombic. It is sufficient that it contains the Coulomb potential.

\section{DERIVATION OF THE SECOND MOMENT SUM-RULE}

Following Ref. [4] we may write the second order BGY hierarchy as follows

$$
\begin{aligned}
\nabla_{\mathbf{r}_{2}} u_{2}(1,2)= & \beta \mathbf{F}_{12}\left[u_{2}(1,2)+1\right]+ \\
& \rho \int d \mathbf{r}_{3}\left[1+u_{2}(1,2)+u_{2}(1,3)+u_{2}(2,3)+u_{3}(1,2,3)\right] \beta \mathbf{F}_{32}- \\
& \rho \int d \mathbf{r}_{3}\left[u_{2}(1,2)+1\right] \beta \mathbf{F}_{32}
\end{aligned}
$$

where $\mathbf{F}_{12}=-\nabla_{\mathbf{r}_{2}} v(1,2)$, with $v(1,2)$ the pair-potential, and the last line in Eq. (5) is for the neutralizing uniform background. We immediately observe that $\int d \mathbf{r}_{3} u_{2}(2,3) \mathbf{F}_{23}=0$ by symmetry.

Multiplying by $\mathbf{r}_{12}=\mathbf{r}_{1}-\mathbf{r}_{2}$ and integrating over $\mathbf{r}_{1}$ we find

$$
\begin{aligned}
\int d \mathbf{r}_{1} \mathbf{r}_{12} \cdot \nabla_{\mathbf{r}_{2}} u_{2}(1,2)= & \int d \mathbf{r}_{1} \mathbf{r}_{12}\left\{\beta \mathbf{F}_{12}+\right. \\
& \left.\rho \int d \mathbf{r}_{3}\left\{u_{2}(1,3)+[\delta(1,3)+\delta(1,2)] u_{2}(2,3) / \rho+u_{3}(1,2,3)\right\} \beta \mathbf{F}_{32}\right\} \\
= & \int d \mathbf{r}_{1} \mathbf{r}_{12}\left\{\int d \mathbf{r}_{3}\left[\rho u_{2}(1,3)+\delta(1,3)\right] \beta \mathbf{F}_{32}+\right. \\
& \left.\rho \int d \mathbf{r}_{3} c_{3}(1 \mid 2,3) \beta \mathbf{F}_{32}\right\},
\end{aligned}
$$

where $s(1,3)=\rho u_{2}(1,3)+\delta(1,3)$ and $c_{3}(1 \mid 2,3)=u_{3}(1,2,3)+[\delta(1,2)+\delta(1,3)] u_{2}(2,3) / \rho$ the excess charge density which does not carry multipoles of any order (See Proposition 2.2 in 
Ref. [1]). Then

$$
\begin{aligned}
\int d \mathbf{r}_{1} \mathbf{r}_{12} \cdot \nabla_{\mathbf{r}_{2}} u_{2}(1,2)= & \int d \mathbf{r}_{1} \mathbf{r}_{12} \int d \mathbf{r}_{3} s(1,3) \beta \mathbf{F}_{32}+ \\
& \rho \int d \mathbf{r}_{3} \beta \mathbf{F}_{32} \int d \mathbf{r}_{1} \mathbf{r}_{12} c_{3}(1 \mid 2,3) .
\end{aligned}
$$

Now we observe that due to the dipole sum-rule [1] the last line in Eq. (77) must vanish, $\mathbf{F}=\mathbf{F}^{s r}+\mathbf{F}^{c}$ can be split into a short-range term, $\mathbf{F}^{s r}$, and a coulombic term, $\mathbf{F}^{c}$, where

$$
\int d \mathbf{r}_{1} \mathbf{r}_{12} \int d \mathbf{r}_{3} s(1,3) \beta \mathbf{F}_{32}^{s r}=\int d \mathbf{r}_{23} \beta \mathbf{F}_{32}^{s r} \int d \mathbf{r}_{13}\left(\mathbf{r}_{13}+\mathbf{r}_{32}\right) s(1,3)=0,
$$

where we used the charge sum-rule and isotropy of the system. This tells us that the result we will find for the second moment is form invariant under the addition to the pair-potential of a generic short-range term. Also, using $\nabla_{\mathbf{r}_{1}}=-\nabla_{\mathbf{r}_{2}}$ and $\int d \mathbf{r}_{1} \ldots=-\int d \mathbf{r}_{2} \ldots$, we find

$$
\int d \mathbf{r}_{1} \mathbf{r}_{12} \cdot \nabla_{\mathbf{r}_{2}} u_{2}(1,2)=3 \int d \mathbf{r}_{1} u_{2}(1,2)=-3 / \rho
$$

where we also used the charge sum-rule. Putting all together, we find

$$
\begin{aligned}
-\frac{3}{\rho} & =\frac{1}{2} \int d \mathbf{r}_{1} \boldsymbol{\nabla}_{\mathbf{r}_{1}}\left(r_{12}^{2}\right) \int d \mathbf{r}_{3} s(1,3) \beta \mathbf{F}_{32}^{c} \\
& =-\frac{1}{2} \int d \mathbf{r}_{1} \boldsymbol{\nabla}_{\mathbf{r}_{2}}\left(r_{12}^{2}\right) \int d \mathbf{r}_{3} s(1,3) \beta \mathbf{F}_{32}^{c} \\
& =\frac{1}{2} \int d \mathbf{r}_{1} r_{12}^{2} \int d \mathbf{r}_{3} s(1,3) \beta \boldsymbol{\nabla}_{\mathbf{r}_{2}} \mathbf{F}_{32}^{c} \\
& =\frac{1}{2} \int d \mathbf{r}_{1} r_{12}^{2} s(1,2) 4 \pi e^{2} \beta
\end{aligned}
$$

where we used the property that $\nabla_{\mathbf{r}_{2}} \mathbf{F}_{32}^{c}=4 \pi e^{2} \delta(3,2)$. And finally we find for the second moment sum-rule

$$
I_{2}=\int d \mathbf{r}_{2} r_{12}^{2} s(1,2)=\frac{3}{2 \pi \rho \beta e^{2}}=\frac{6}{k_{D}^{2}}
$$

where $\lambda_{D}=k_{D}^{-1}=\left(4 \pi \rho \beta e^{2}\right)^{-1 / 2}$ is the Debye-Hückel screening length. 


\section{DERIVATION OF THE FOURTH MOMENT SUM-RULE}

Starting from Eq. (5) we multiply by $r_{12}^{2} \mathbf{r}_{12}$ and integrate over $\mathbf{r}_{1}$ to get

$$
\begin{aligned}
\int d \mathbf{r}_{1} r_{12}^{2} \mathbf{r}_{12} & \cdot \nabla_{\mathbf{r}_{2}} u_{2}(1,2)=\int d \mathbf{r}_{1} r_{12}^{2} \mathbf{r}_{12}\left\{\beta \mathbf{F}_{12}+\right. \\
& \left.\rho \int d \mathbf{r}_{3}\left\{u_{2}(1,3)+[\delta(1,3)+\delta(1,2)] u_{2}(2,3) / \rho+u_{3}(1,2,3)\right\} \beta \mathbf{F}_{32}\right\} \\
= & \int d \mathbf{r}_{1} r_{12}^{2} \mathbf{r}_{12}\left\{\int d \mathbf{r}_{3}\left[\rho u_{2}(1,3)+\delta(1,3)\right] \beta \mathbf{F}_{32}+\rho \int d \mathbf{r}_{3} c_{3}(1 \mid 2,3) \beta \mathbf{F}_{32}\right\} \\
= & \int d \mathbf{r}_{1} r_{12}^{2} \mathbf{r}_{12} \int d \mathbf{r}_{3} s(1,3) \beta \mathbf{F}_{32}+\rho \int d \mathbf{r}_{3} \beta \mathbf{F}_{32} \int d \mathbf{r}_{1} r_{12}^{2} \mathbf{r}_{12} c_{3}(1 \mid 2,3) .
\end{aligned}
$$

Note that splitting again into a short-range term and the Coulomb one we find for the first term on the right hand side of Eq. (12)

$$
\begin{aligned}
\int d \mathbf{r}_{3} \beta \mathbf{F}_{32}^{s r} \int d \mathbf{r}_{1} r_{12}^{2} \mathbf{r}_{12} s(1,3) & =-\int d \mathbf{r}_{32} \beta v^{s r}(3,2) \int d \mathbf{r}_{12} \nabla_{\mathbf{r}_{12}}\left[r_{12}^{2} \mathbf{r}_{12} s(1,3)\right], \\
& =\int d \mathbf{r}_{32} \beta v^{s r}(3,2) \int d \mathbf{S}_{12} r_{12}^{2} \mathbf{r}_{12} s(1,3)=0
\end{aligned}
$$

Since $s(1,3)=s\left(\left|\mathbf{r}_{32}+\mathbf{r}_{21}\right|\right)$ decays exponentially fast as $r_{12}$ tends to infinity and the surface integral is over a sphere centered on $r_{12}=0$ and with an infinite radius. The same holds for the second term on the right hand side of Eq. (12).

This proves that the result we will find is independent from the addition of a short-range part to the Coulomb pair-potential.

Now we observe that

$$
\begin{aligned}
\int d \mathbf{r}_{1} r_{12}^{2} \mathbf{r}_{12} \int d \mathbf{r}_{3} s(1,3) \beta \mathbf{F}_{32}^{c} & =\frac{1}{4} \int d \mathbf{r}_{1} \boldsymbol{\nabla}_{\mathbf{r}_{1}}\left(r_{12}^{4}\right) \int d \mathbf{r}_{3} s(1,3) \beta \mathbf{F}_{32}^{c} \\
& =-\frac{1}{4} \int d \mathbf{r}_{1} \boldsymbol{\nabla}_{\mathbf{r}_{2}}\left(r_{12}^{4}\right) \int d \mathbf{r}_{3} s(1,3) \beta \mathbf{F}_{32}^{c} \\
& =\frac{1}{4} \int d \mathbf{r}_{1} r_{12}^{4} \int d \mathbf{r}_{3} s(1,3) \beta \boldsymbol{\nabla}_{\mathbf{r}_{2}} \mathbf{F}_{32}^{c} \\
& =\frac{1}{4} \int d \mathbf{r}_{1} r_{12}^{4} s(1,2) 4 \pi e^{2} \beta .
\end{aligned}
$$

And also using integration by parts

$$
\begin{aligned}
\int d \mathbf{r}_{1} r_{12}^{2} \mathbf{r}_{12} \cdot \nabla_{\mathbf{r}_{2}} u_{2}(1,2) & =\int d \mathbf{r}_{1} \nabla_{\mathbf{r}_{12}}\left(r_{12}^{2} \mathbf{r}_{12}\right) u_{2}(1,2) \\
& =5 \int d \mathbf{r}_{1} r_{12}^{2} u_{2}(1,2) \\
& =-\frac{15}{2 \pi \rho^{2} \beta e^{2}}
\end{aligned}
$$


where in the last equation we used the main result of previous section for the second moment condition (11).

In this case $\int d \mathbf{r}_{1} d \mathbf{r}_{3} \beta \mathbf{F}_{32} r_{12}^{2} \mathbf{r}_{12} c_{3}(1 \mid 2,3) \neq 0$ and we may recognize in such a term the one giving rise to the isothermal compressibility in Eq. (21).

Putting together Eqs. (12), (13), (17), (20), and (22) we should reach the following fourth moment result

$$
I_{4}=\int d \mathbf{r}_{2} r_{12}^{4} s_{2}(1,2)=\frac{15}{2 \pi^{2} \rho^{2} \beta^{2} e^{4}} \frac{\chi_{T}^{0}}{\chi_{T}}=\frac{120}{k_{D}^{4}} \frac{\chi_{T}^{0}}{\chi_{T}},
$$

where $\frac{1}{\chi_{T} \rho}=\left.\frac{\partial P}{\partial \rho}\right|_{T}$ is the isothermal compressibility and $\chi_{T}^{0}=\beta / \rho$ the one of the ideal gas. As already stressed this result is independent from the addition of a short-range term to the Coulomb pair-potential.

Then we should be able to prove that

$$
\begin{aligned}
1-\frac{\chi_{T}^{0}}{\chi_{T}} & =\frac{\rho \int d \mathbf{r}_{3} \beta \mathbf{F}_{32}^{c} \int d \mathbf{r}_{1} r_{12}^{2} \mathbf{r}_{12} c_{3}(1 \mid 2,3)}{\int d \mathbf{r}_{1} r_{12}^{2} \mathbf{r}_{12} \cdot \nabla_{\mathbf{r}_{2}} u_{2}(1,2)} \\
& =\frac{\rho}{5} \frac{\int d \mathbf{r}_{3} \beta \mathbf{F}_{32}^{c} \int d \mathbf{r}_{1} r_{12}^{2} \mathbf{r}_{12} c_{3}(1 \mid 2,3)}{\int d \mathbf{r}_{1} r_{12}^{2} u_{2}(1,2)} \\
& =-\frac{2 \pi \rho^{3} \beta^{2} e^{2}}{15} \int d \mathbf{r}_{32} d \mathbf{r}_{12} c_{3}(1 \mid 2,3) r_{12}^{2} \mathbf{r}_{12} \cdot \nabla_{\mathbf{r}_{32}} v^{c}(3,2) \\
& =-\frac{2 \pi \rho^{3} \beta^{2} e^{2}}{9} \int d \mathbf{r}_{32} d \mathbf{r}_{12} c_{3}(1 \mid 2,3) r_{12}^{2} \mathbf{r}_{32} \cdot \nabla_{\mathbf{r}_{32}} v^{c}(3,2),
\end{aligned}
$$

where in the last equality we used $\mathbf{r}_{12}=\mathbf{r}_{13}+\mathbf{r}_{32}, \nabla_{\mathbf{r}}\left(r^{2} \mathbf{r}\right)=5 r^{2}, r^{2} \nabla_{\mathbf{r}}(\mathbf{r})=3 r^{2}$, and integration by parts. This will be done in the next section.

\section{COMPRESSIBILITY SUM-RULE}

From the virial theorem follows that the pressure estimator can be written as follows [10],

$$
\beta P=\rho-\frac{\beta \rho^{2}}{6} \int d \mathbf{r} u_{2}(r) \mathbf{r} \cdot \nabla_{\mathbf{r}} v^{c}(r)
$$

So that

$$
1-\frac{\chi_{T}^{0}}{\chi_{T}}=1-\left.\beta \frac{\partial P}{\partial \rho}\right|_{T}=\frac{\beta}{6} \int d \mathbf{r} \frac{\partial \rho^{2} u_{2}(r)}{\partial \rho} \mathbf{r} \cdot \nabla_{\mathbf{r}} v^{c}(r),
$$

We then see that Eq. (22) can be obtained using an analysis similar to the one of Vieillefosse et al. [16], thus finding

$$
\frac{\partial \rho^{2} u_{2}\left(r_{32}\right)}{\partial \rho}=-\frac{4 \pi \rho^{3} \beta e^{2}}{3} \int d \mathbf{r}_{12} r_{12}^{2} c_{3}(1 \mid 2,3) .
$$


We then see how $\chi_{T}$ is the isothermal compressibility of a plasma with a Coulomb interaction pair-potential among the particles.

\section{CONCLUSIONS}

We determined the first three (even) structure factor moment sum-rules (44), (11), (21) for a three-dimensional Jellium with the particles interacting with a pair-potential that is the sum of the Coulomb potential $e^{2} / r$ and a short-range term with either a finite range or decaying exponentially fast at large $r$. We found that they are all invariant in form respect to the addition of the short-range term. Moreover our derivations of the sum-rules are different and simpler than the ones already found in the literature (as described in the review of Ph. Martin [1]). This strategy carry us to the determination of an compressibility sum-rule (24)-(25) in agreement with the one of Vieillefosse [16].

When studying common matter, whose constituents are made of charged particles, the Coulomb interaction plays a special role, ruling the fundamental correlation sum-rules. What really matter is the long-range nature of the Coulomb interaction and the short-range details do not have an influence on the statistical behaviors of the many-body correlations. This allows to use different models for the charges behavior at short-range where we may have some sort of indeterminacy in the description of the point-wise constituents particles microscopic character. All these models will have the same macroscopic behavior.

We could for example apply our general setting to the particular case of charged hardspheres, when the short-range term is just a hard-core repulsion of a certain diameter. This is just one of the commonly used short-range regularization employed in a two-componentplasma (TCP) with particles of opposite charges [13, 17, 18] that would otherwise collapse one over the other. Moreover the hard-core model has been historically the favorite playground in statistical mechanics as it represents the simplest model of many-body systems of interacting particles.

In a recent work Das, Kim, and Fisher [11, 12] found out, through finely discretized grand canonical Monte Carlo simulations, that in the Restricted Primitive Model (RPM) of an electrolyte [17, 18], the second- and fourth-moment charge-charge sum-rules, typical for ionic fluids, are violated at criticality. For a 1:1 equisized charge-symmetric hard-sphere electrolyte their grand canonical simulations, with a new finite-size scaling device, confirm 
the Stillinger-Lovett second-moment sum-rule except, contrary to current theory [19], for its failure at the critical point $\left(T_{c}, \rho_{c}\right)$. Furthermore, the $k^{4}$ term in the charge-charge correlation or structure factor $S_{Z Z}(k)$ expansion is found to diverge like the compressibility when $T \rightarrow T_{c}$ at $\rho_{c}$. These findings are in evident disagreement with available theory for charge-symmetric models and, although their results are qualitatively similar to behavior expected for charge-asymmetric systems [19], even a semi-quantitative understanding has eluded them. Our present study could be a first step towards an explanation of such puzzling behavior. Even if, as pointed out in Ref. [14], from the work of Santos and Piasecki [15] follows that the Ursell functions of any order are likely to have a long-range behavior on a critical point, thus violating our exponential clustering working-hypothesis.

The zeroth-, second-, and fourth-moment sum-rules are rigorously derived starting from the Born-Green-Yvon equations and the exponential clustering hypothesis by Suttorp and van Wonderen [20 22] for a thermodynamically stable ionic mixture made of point-wise particles of charges all of the same sign immersed in a neutralizing background, the Jelliummixture. Our results show that the addition of a hard-core, or more generally any finite-range or exponentially decaying contribution to the pair-potential, to the particles, which would be necessary in order to make thermodynamically stable the system of Suttorp and van Wonderen for mixtures with particles of opposite charges, does not change the form of the first two three moments of the structure factor of the one-component Jellium.

It is still an open problem the extension of our study to the more general case of a mixture. A semi-heuristic derivation has recently been carried out [13, 14] showing that the addition of the short-range term should not play any role at the level of the first three (even) structure factor moments for a neutral TCP without the background. Strictly speaking, in these derivations we had to use results that are only rigorously valid in the Debye regime, like the local neutrality of the homogeneous system. Our present rigorous result confirms this scenario, at least in the weak coupling limit. Another interesting project is to generalize these sum-rule results to the case of Jellium living in curved surfaces [23 26] . In these cases the system can be mapped in an equivalent flat Jellium interacting with an external potential generated by the curvature of the surface in which the particles live. Another interesting extension of our work consists in studying the case in which the short-range pair-potential decays at large distances as an inverse power $s$ of the distance, in which case the decay of correlations is also always algebraic, with the only exception of $s=\nu-2$ with $\nu$ the 
space dimension [9]. In this case we must drop the exponential clustering hypothesis and our present derivation is not valid anymore.

\section{ACKNOWLEDGMENTS}

[1] P. A. Martin Rev. Mod. Phys. 60, 1075 (1988).

[2] P. Debye and E. Hückel Phys. Z. 9, 185 (1923).

[3] F. H. S. Jr. and R. Lovett J. Chem. Phys. 49, 1991 (1968).

[4] P. A. Martin and C. Gruber J. Stat. Phys. 31, 691 (1983).

[5] P. Vieillefosse J. Stat. Phys. 41, 1015 (1985).

[6] D. Pines and P. Nozières, The Theory of Quantum Liquids (Benjamin, New York, 1966) p. 219.

[7] P. Vieillefosse and J. P. Hansen Phys. Rev. A 12, 1106 (1975).

[8] M. Baus J. Phys. A 11, 2451 (1978).

[9] A. Alastuey and P. A. Martin J. Stat. Phys. 39, 405 (1985).

[10] J.-P. Hansen and I. R. McDonald, Theory of Simple Liquids, 2nd ed. (Academic Press, London, 1986).

[11] S. K. Das, Y. C. Kim, and M. E. Fisher Phys. Rev. Lett. 107, 215701 (2011).

[12] S. K. Das, Y. C. Kim, and M. E. Fisher J. Chem. Phys. 137, 074902 (2012).

[13] A. Alastuey and R. Fantoni J. Stat. Phys. 163, 887 (2016).

[14] R. Fantoni Physica A 477C, 187 (2017).

[15] A. Santos and J. Piasecki Mol. Phys. 113, 2855 (2015).

[16] P. Vieillefosse and M. Brajon J. Stat. Phys. 55, 1169 (1989).

[17] R. Fantoni and G. Pastore Europhys. Lett. 101, 46003 (2013).

[18] R. Fantoni and G. Pastore Phys. Rev. E 87, 052303 (2013).

[19] G. Stell J. Stat. Phys. 78, 197 (1995).

[20] L. G. Suttorp and A. J. van Wonderen Physica A 145, 533 (1987).

[21] A. J. van Wonderen and L. G. Suttorp Physica A 145, 557 (1987).

[22] L. G. Suttorp J. Phys. A: Math. Theor. 41, 495001 (2008). 
[23] R. Fantoni, B. Jancovici, and G. Téllez J. Stat. Phys. 112, 27 (2003).

[24] R. Fantoni and G. Téllez J. Stat. Phys. 133, 449 (2008).

[25] R. Fantoni J. Stat. Mech. , P04015 (2012).

[26] R. Fantoni J. Stat. Mech. , P10024 (2012). 